\title{
Fuzzy Logic based High Speed Network Congestion Control
}

\author{
Ajay Kumar \\ Student M. Tech (CSE), Panchkula Engineering \\ College \\ Mauli, Panchkula, Haryana \\ India
}

\author{
Amit Jain \\ Associate Professor's Panchkula Engineering \\ College \\ Mauli, Panchkula, Haryana, India
}

\begin{abstract}
In this paper, we describe the formatting guidelines for IJCA Journal Submission. Congestion is the problem that occurs due to saturation of network resources. Still the implementation of traditional congestion control algorithms such as OSI Layer 4 Transmission Control Protocol/ Internet Protocol (TCP / IP), due Objected Oriented congestion remains a critical issue in Local Network, ATM networks and SONET. Fuzzy Logic is applied to resolve the network traffic control problem as medium of networks are too difficult using traditional control system theory. Fuzzy Logic based congestion control good result the traditional methods in various cases. It is the first time that an explicit rate-based congestion control system designed with the fuzzy logic control is proved globally asymptotically stable. This Paper is a review of Fuzzy Logic and Neural-Fuzzy based techniques that applied to deal with congestion. Fuzzy Logic based Congestion Controller is a model free controller that utilizes qualitative reasoning to implement non-linear control functions efficiently.
\end{abstract}

\section{Keywords}

Congestion control, Fuzzy Logic, ATM Networks, NeuralFuzzy Networks, Fuzzy Inference Systems (FIS)

\section{INTRODUCTION}

Congestion is the problem is said to be occurred when the Source transmission rate exceeds the data handling capacity of the network. The degradation in performance is observed. Congestion is said to be occurred if network resources are not efficiently managed. If the source delivers at a rate higher than service rate of queue, then the size of queue will grow. If the size of queue is finite, then packets will experience delay \& losses will occur. End users experience delay in delivery of packets, even loss of packets also [1]. Networks that try to send more data than network capacity, lead to data loss and excessive delays in packet delivery [2]. Due to unpredictable fluctuations and burst of traffic flow in networks, congestion occurs frequently [3]. The problem of congestion has become more severe due to increased use of internet for high speed, delay sensitive applications with variety of Quality of Service (QoS) requirements [4]. Congestion control is a process in which networks adjusts the amount of input data, so that the Quality of Service (QoS) is not degraded and simultaneously try to utilize the network resources efficiently [2]. The basic idea of fuzzy control is to Design of fuzzy logic control involves selection of suitable mathematical representations for t-norms, s-norm, defuzzification operator, fuzzy implications and shapes of membership functions [3]. Fuzzy Logic is a logical system which is an extension of multi-valued logic. It was introduced by Zadeh and firstly applied by Mamdani to design control systems [4].
Fuzzy Logic Controllers are seen as an alternative to design feedback controllers to deal with congestion. As Computer Networks have a large distributed area, so they are highly non-linear, time varying and. Modelling of such networks is very difficult [1]. As the complexity increases it becomes highly difficult to model a system. [5]. Fuzzy Logic Controllers do not need formal models of control systems. The control algorithm is a set of rules [1].

Its architecture is expected to provide a mechanism for protecting individual flows from congestion, and introduces its own queue management and scheduling algorithms. In [6] it is speculated whether a virtual circuit model should be adopted (i.e. abandonment of IP), as proposed in ATM and ST-II protocol. Debate is still at an early stage, but the approach to congestion control should be based on a congestion control framework and appropriate control techniques (the same comments apply for differentiated services in the Internet).

\section{FUZZY LOGIC APPLICATION FOR CONGESTION CONTROL}

A network system is a large distributed complex system, with difficult often highly non-linear, time varying and chaotic behavior. Computational Intelligence (CI) [7], [8] is an area of fundamental and applied research involving numerical information processing (in contrast to the symbolic information processing techniques of Artificial Intelligence (AI)). Nowadays, CI research is very active and consequently its applications are appearing in some end user products. The definition of CI can be given indirectly by observing the exhibited properties of a system that employs CI components [7]:

"A system is computationally intelligent when it: deals only with numerical (low-level) data, has a pattern recognition component, and does not use knowledge in the AI sense; and additionally, when it (begins to) exhibit

- Computational adaptively, computational fault tolerance;

- $\quad$ speed approaching human-like turnaround;

- Error rates that approximate human performance.

Fuzzy Logic Controllers (FLCs) may be viewed as substitute, non-conventional way of designing feedback controllers where it is convenient and effective to build a control algorithm without relying on formal models of the controlled system and control theoretic tools. The control algorithm is encapsulated as a set of commonsense rules. FLCs have been applied successfully to the task of controlling systems for which analytical models are not easily obtainable or the model itself, if available, is too complex and highly nonlinear. 
Therefore, the researchers who applied the computational intelligence methods to congestion control problem mostly looked at predictive congestion control schemes. In general, the schemes observe the short term behavior of a link to estimate the future of cell arrivals in order to predict the onset of congestion and take proactive measures to prevent its occurrence. Liu and Douligeris [11] have proposed a combination system consisting of a leaky bucket and a fuzzy logic cell rate controller.

Jensen [12] has proposed a fuzzy system for controlling the transmission rate of sources to protect links against overload in the case of connections exceeding their negotiated traffic parameters. Cheng and Chang [13], [14] combine connection admission control (CAC) and congestion control mechanisms. The congestion control mechanism sends back coding rate control signals to video and audio sources, and congestion control signals to data sources to adjust the cell transmission rate of the sources, and subsequently the traffic density at the Switches.

Pitsillides et al. [15], [16], and Qiu [17] have proposed congestion control schemes which operate under similar principles. The schemes, by measuring the queue length and queue growth rates at the output buffer of a switch, attempt to estimate the future behavior of the queue, and send explicit rate control signals to the traffic sources to avoid or alleviate congestion. The explicit rate control signals are calculated periodically by fuzzy inference engines located in the switches, and sent to the traffic sources in resource management (RM) cells. The scheme of Pitsillides et al. is used in Fuzzy Explicit Rate Marking (FERM) algorithm. They have analyzed its performance in detail regarding fairness, responsiveness, resource utilization and cell loss in LAN and WAN environments. The scheme has been further refined (FERM2) and as an adaptive scheme which has selftuning capabilities (A-FERM). A detailed overview of FERM2 is presented in the next section. Note

\section{FERM2 CONGESTION CONTROL ALGORITHM}

In this section the operation of FERM2 explicit rate congestion control scheme is summarized. FERM2 is very similar to FERM [16] and can be considered as a further refinement of the original scheme. The main difference between the two schemes is in the former one the desired queue length is implicit, in the later one it is set by a higher level control module to provide more dynamic resource utilization across the switches constituting the virtual connection. Figure 1 shows the block diagram FERM2.
Similar to the original algorithm, FERM2 also uses the following five parameters.

$\begin{array}{ll}\text { Parameter } & \text { Definition } \\ \text { PCR } & \text { Peak cell rate } \\ \text { ICR } & \text { Initial cell rate } \\ \text { AIR } & \text { Additive increase rate } \\ \text { MCR } & \text { Minimum cell rate } \\ \text { Nfp } & \text { Control interval }\end{array}$

Overall operation of the scheme is compliant with the ATM Forum Traffic Management Specification, Version 4. Cell rates of data sources are adjusted by Explicit Rate (ER) information carried by Resource Management (RM) cells. RM cells are periodically generated by traffic sources, transmitted towards the destination end systems, and initial ER information is set by the ICR. The destination end systems bounce the RM cells back to the sources. During the return path, when a RM cell passes through an ATM switch, its ER value is examined and possibly modified. A data source, upon receiving a RM cell, adjusts its cell rate based on the value contained in the RM cell's ER field. If the ER field contains a rate bigger than PCR, the cell rate is set to PCR. Similarly, the cell rate is set to MCR for the ER values less than MCR

The scheme provides the ER to all the active VCs at all the time so that congestion and undesired resulting behavior can be avoided. The scheme does not need to keep the state of current VC connections sharing the same semi-static VP at the switch.

\subsection{Rule base design}

The selection of rule base is based on the designer's experience and beliefs on how the system should behave. Design of rule base is two -fold: First, the linguistic rules ("surface structure") are set; afterwards, membership functions of the linguistic values ("deep structure") are determined. The trade-off involving the design of the rule base is to have a set of minimum number of linguistic rules representing the control surface with sufficient accuracy to achieve an acceptable performance. Recently, in the fuzzy control literature,

Some formal techniques for obtaining a rule base by using Artificial Neural Networks or Genetic Algorithms have appeared. Nevertheless, the conventional trial and error approach under the guidance of some design rules of thumb [40] can be referred or a discussion of these) have been used in this study.

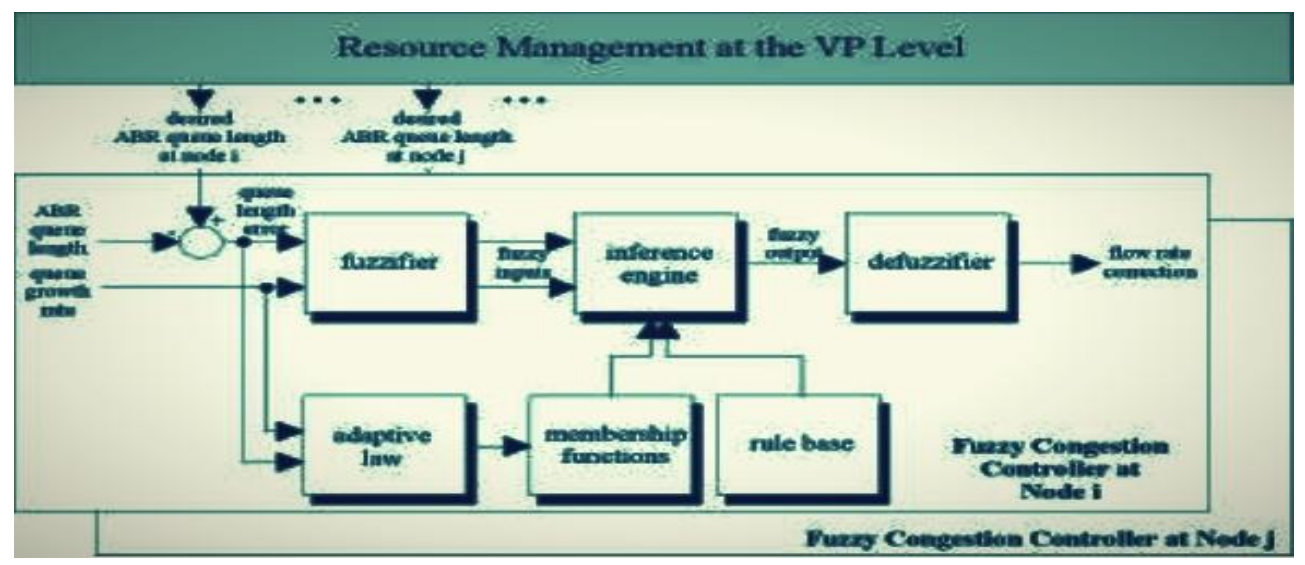

Figure 1. Block diagram of the Fuzzy Congestion Controller of the FERM2 scheme 
Usually, to define the linguistic rules of a fuzzy variable, Gaussian like, triangular or trapezoidal shaped membership functions are used. Selection of Gaussian like membership functions leads to smoother control surfaces. Then, the rule base is fine-tuned by observing the progress of simulation, such as cell loss occurrences and demand versus throughput curves. The tuning can be done with different objectives in mind. For example, any gain in throughput must be traded off by a possible increase in the delay experienced at the terminal queues. However, since the tuning of the fuzzy rules is intuitive, and can be related in simple linguistic terms with user's experience, it should be a straightforward matter to

Achieve an appropriate balance between a tolerable end-toend delay, and the increase in throughput. The tuning objective can be based on a desired optimization criterion, for example, a tradeoff between maximization of throughput with minimization of end-to-end delay experienced by the users. The set of linguistic rules shown below in Table 1 define the control surface of the FCC:

if $\mathrm{ABR}$ queue length is too short and queue is decreasing fast then increase flow rate sharply

if $\mathrm{ABR}$ queue length is too short and queue is decreasing slowly then increase flow rate moderately

if $\mathrm{ABR}$ queue length is too short and queue length is not changing then increase flow rate moderately

if ABR queue length is too short and queue is increasing slowly then decrease flow rate moderately

if $\mathrm{ABR}$ queue length is too short and queue is increasing fast then decrease flow rate moderately

if ABR queue length is acceptable and queue is decreasing fast then increase flow rate moderately

if ABR queue length is acceptable and queue is decreasing slowly then increase flow rate moderately

if ABR queue length is acceptable and queue length is not changing then do not change flow rate

if $\mathrm{ABR}$ queue length is acceptable and queue is increasing slowly then decrease flow rate moderately

if $\mathrm{ABR}$ queue length is acceptable and queue is increasing fast then decrease flow rate moderately

if $\mathrm{ABR}$ queue length is too high and queue is decreasing fast then do not change flow rate

if $\mathrm{ABR}$ queue length is too high and queue is decreasing slowly then do not change flow rate

if $\mathrm{ABR}$ queue length is too high and queue length is not changing then decrease flow rate moderately if $\mathrm{ABR}$ queue length is too high and queue length is increasing slowly then decrease flow rate sharply

if $\mathrm{ABR}$ queue length is too high and queue length is increasing fast then decrease flow rate sharply

Table 1. Set of linguistic rules defining the control surface of the FCC

The control surface is shaped by the rule base and the linguistic values of the linguistic variables. By observing the progress of simulation, and modifying the rules and definitions of the linguistic values, FCC can be tuned to achieve better server utilization, and lower cell loss coupled with minimal end-to-end cell delay.

\subsection{DESIGN CHALLENGES}

The linguistic rules which determine the actions to be taken by the FLCs can sometimes pose challenges to the designers. Traditionally, the rules encapsulate the expert's experience or belief about the necessary control actions taken. Cheng and Chang [13], [14] have a genetic algorithms to obtain linguistic rules from the operational data. Another challenge is, usually the rules are static: they do not change during the operation of the system. Naturally, this can lead to suboptimal control actions to be taken if system dynamics change in time. The solution to this problem is to use adaptive methods to modify a set of parameters which are used to define the linguistic rules in real-time. Sugeno [18] has proposed a method for adaptive tuning of linguistic rules of a FLC. In his method, the controlled variable which determines the output action defined in the linguistic rules are chosen as polynomial expressions of some state variables whose coefficients are modified by adaptive techniques. This technique can be used for controlling very complex systems and has been successfully demonstrated by Sugeno to control the flight of a helicopter. For an illustrative example of this very interesting approach see [19] and [20]. Hu, Petr and Braun [21] have used this method to design an adaptive fuzzy congestion control scheme. In their approach, the level of network congestion is monitored through the queue length at the output buffer of the switch, with the control target being set at a desired queue length. Sekercioglu and Pitsillides have also designed an adaptive control scheme (A-FERM).

\section{SIMULATIVE EVALUATION}

The authors have done extensive simulations on a representative ATM network (Figure 3) and have compared the performance of FERM against enhanced proportional rate control algorithm (EPRCA). The results of this study has been reported in [16]. FERM2 yields yet better throughput results than FERM in an overloaded network for both LAN and WAN networks (Figure 4 and Figure 5). 


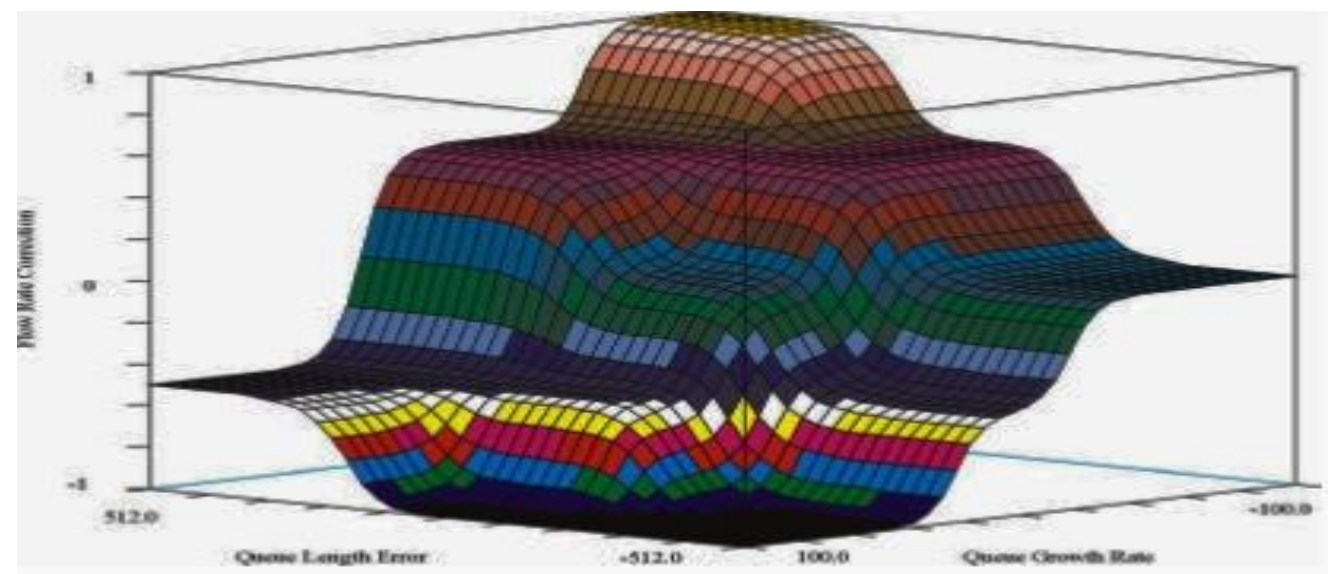

Figure 2. Control surface of the Fuzzy Congestion Controller

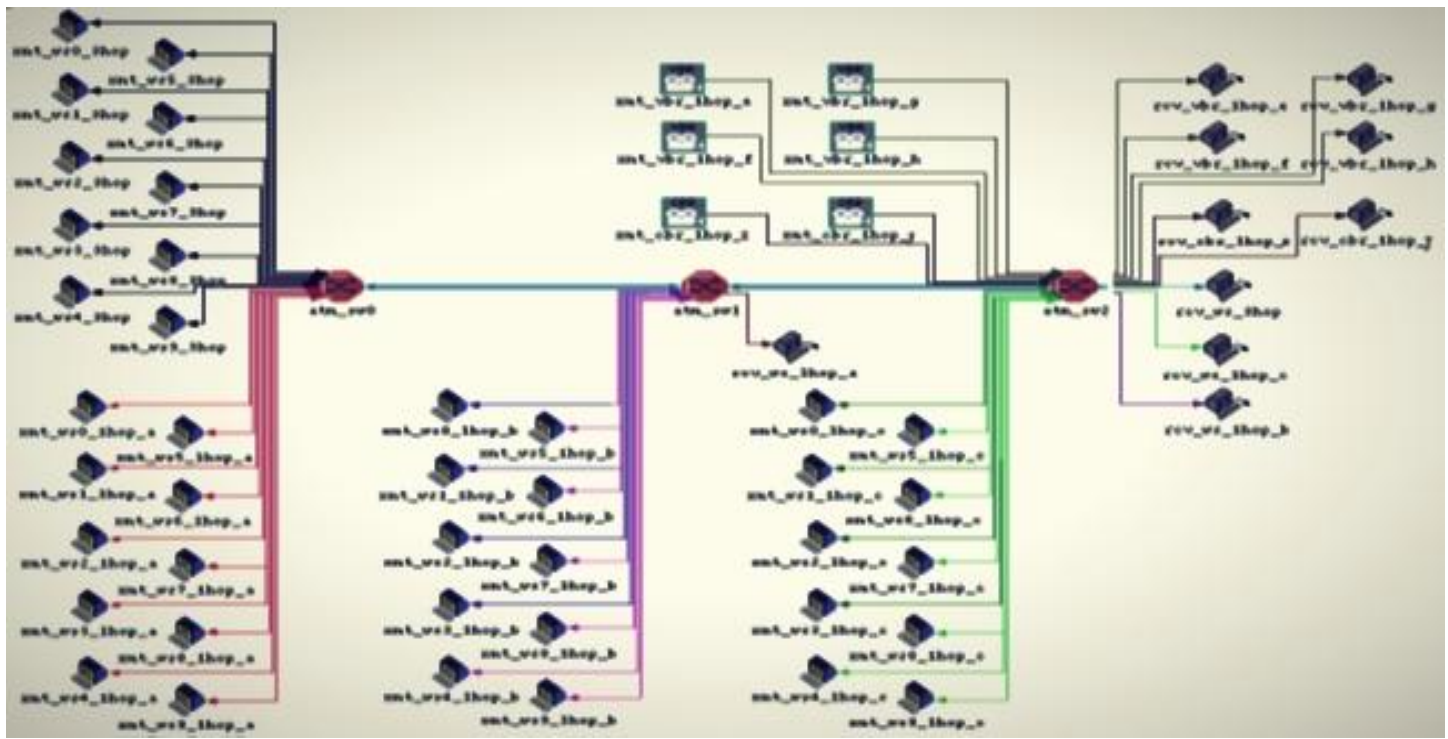

Figure 3. ATM network model used for performance analysis of FERM and FERM2 algorithm. Same network Configuration has been used for the simulation of ATM WAN backbone and ATM LAN backbone except that the distances between switches have been set to $1500 \mathrm{~km}$ and $10 \mathrm{~km}$ for WAN and LAN simulations respectively. All traffic (except 1hop (b) traffic) leaving ATM switch 2 travels to a fourth ATM switch and distributed. Since no cell buffering occurs at this switch, it has not been included into the simulation model. The speed of all links have been considered as $155 \mathrm{Mb} / \mathrm{s}$

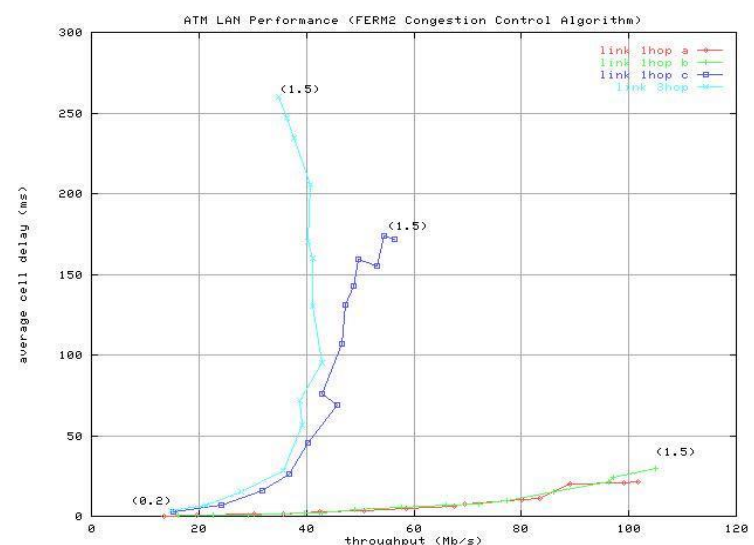

Figure 4. Plot of average end-to-end ABR cell delay vs. useful throughput of simulated ATM LAN under FERM2 congestion control. The graph has been produced by varying the offered link loads generated by the ABR traffic sources from $20 \%$ to $150 \%$ of the link capacities

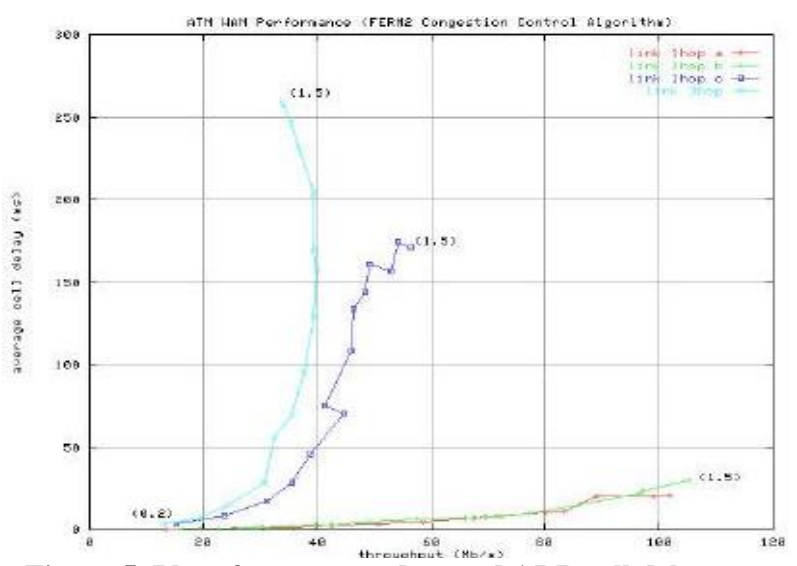

Figure 5. Plot of average end-to-end ABR cell delay vs. useful throughput of simulated ATM WAN under FERM2 congestion control. The graph has been produced by varying the offered link loads generated by the ABR traffic sources from $20 \%$ to $150 \%$ of the link capacities 
The following plots show the time evolution of the Explicit Rate, as calculated by FCC, for the case of a LAN (Figure 6) and a WAN network (Figure 8). The other two figures show the time evolution of the queue length for both LAN (Figure delay products (WAN), as opposed to the excellent controlled system performance for the case of very small propagation delay (LAN). Nevertheless even for the WAN case, the network system is well controlled and the network losses and retransmissions are limited. Note that the distances between switches are set at $1500 \mathrm{~km}$, with a maximum end-to-end round trip delay around $30 \mathrm{msec} @ 6000 \mathrm{kms}$; compare with the 2.6 msec time it takes to fill or empty a buffer of 1000 cells@155 Mbits/sec.

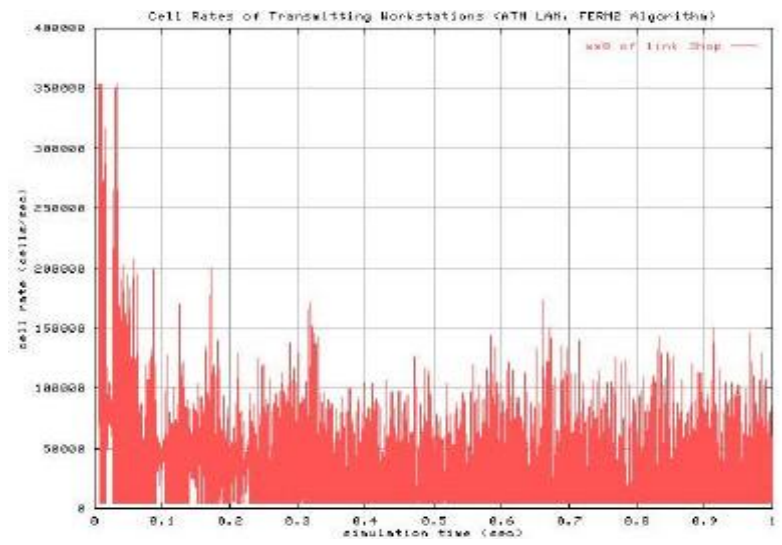

Figure 6 Time evolution of the Explicit Rate for the case of the LAN; calculated by the FCC

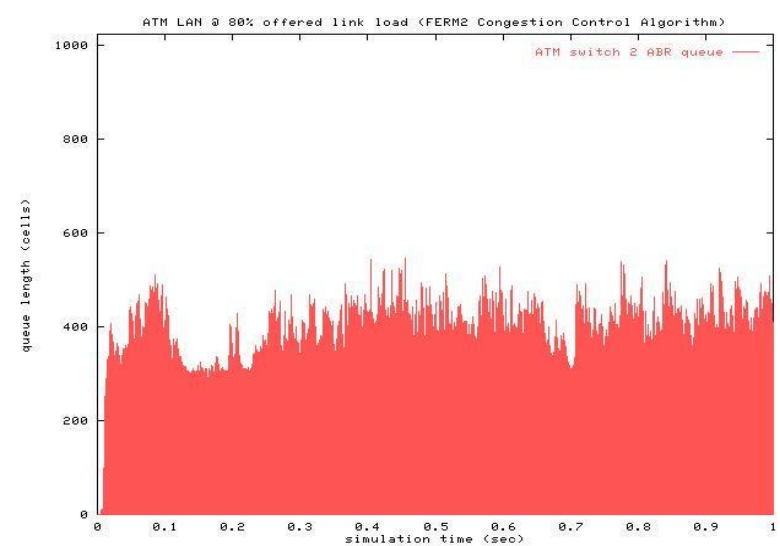

Figure 7. Time evolution of the queue length for the case of a LAN. Note that the reference value is set at 500 cell places

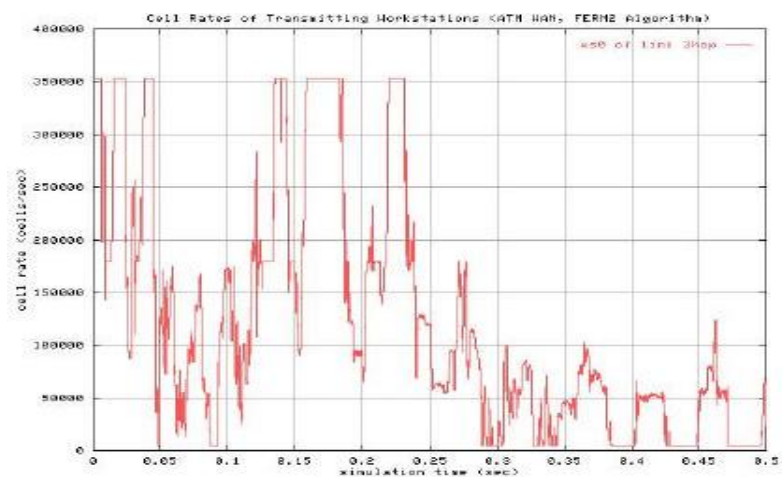

Figure 8. Time evolution of the Explicit Rate for the case of the WAN; calculated by the FCC

\section{CONCLUSIONS AND RECOMMENDATIONS}

In the "fight" against congestion, despite the research efforts spanning a few decades and the large number of different schemes proposed, there are no universally acceptable solutions (a control strategy, a control system, or a "package" of control solutions). Congestion control remains a critical issue and a high priority, especially given the growing size, demand, and speed (bandwidth) of the increasingly integrated services network. There is no doubt that we will see more and more use of these techniques, including their use in the IP world. We also expect that, as in other commercial products, CI techniques will finally make it into real products in this area, and we expect with tremendous success.

Of course many challenges to the control of congestion remain unresolved (after all the network control system is one of the most, if not the most, complex control system that man ever made-it is complex as well as large-scale). Challenges, include:

- Get agreement on a structured approach to congestion control for the network. Control theoretic concepts and techniques have an essential role to play.

- Engineer the network system with the network control system together in order to add another degree of flexibility.

- Deploy these control systems in the large scale, geographically distributed network system. Theoretical advances in handling large scale complex systems are required, including decomposition and organization of controls (possibly hierarchical, multilayer, and multilevel)

- Globally optimize the overall network objectives.

- Develop a framework for the evaluation of the performance of the controlled systems for different control solutions. The framework will possibly have to be simulative (a Common Simulative Framework, CSF). The CSF will have to define and include a number of predefined scenario of test loads, test networks and controlled system performance indices (these will include the indices discussed in [21], such as fairness, steady state response, transient response, rise time, settling time, and controller properties, as e.g. stability, robustness, efficiency, implementation complexity, ease of tuning, scalability, internetworking with other schemes, policing of connections, ...).

In conclusion, there is a real challenge in the control of congestion in communication networks, especially the ones supporting video, voice and data applications simultaneously. Computational Intelligence techniques are expected to play a central role, especially in the large scale, geographically distributed network systems. Hybrids are also expected to supplement these techniques and prove useful, especially in optimizing the overall network objectives.

\section{REFERENCES}

[1] Andreas Pitisillides and Ahmet Sekerciouglu "Fuzzy Logic based Congestion Control" http://Citeseerx.ist.psu.edu/viewdoc/summery?doi=10.1. 1.69 .4324

[2] A. Benzaouia, F. Mesquine and S. El Faiz "Rate-Based Flow Fuzzy Controller for Communication Systems" 
Proceedings of 1st African Control Conference, Cape Town, South Africa, 2003, pp. 120-123.

[3] Andreas Pitsillides, Y. Ahmet Sekercioglu and Gopalakrishnan Ramamurthy "Effective Control of Traffic Flow in ATM Networks Using Fuzzy Explicit Rate Marking (FERM)" IEEE Journal on selected areas in Communications, Vol 15, NO 2, 1997, pp.209-225.

[4] C. Chrysostomou, A. Pitsillides, Y. A. Sekercioglu "Fuzzy Explicit Marking: A UnifiedCongestion Controller for Best-Effort and Diff- Serv Networks" Journal on Computer Networks, 2009, pp.650-667.

[5] Shie-Jue Lee, Chun-Liang Hou "A Neural-Fuzzy System for Congestion Control in ATM Networks" IEEE Transactions on Systems, MAN and Cybernetics, Vol 30,No 1, 2000, pp.2

[6] R. Braden, D. Clark, S.Shenker, Integrated services in the internet architecture: an overview, RFC 1633, July 1994.

[7] J. C. Bezdek, What is Computational Intelligence?, in Computational Intelligence: Imitating Life, edited by J.M. Zurada, R.J. Marks II and C.J. Robinson, IEEE Press, pp. 1-12, 1994.

[8] W.Pedrycz, Computational Intelligence: An Introduction, CRC Press, 1998.

[9] Special issue on Computational Intelligence, IEEE Journal on Selected Areas in Communications (JSAC), Volume 15, Issue 2, February 1997.

[10] B. Azvine (chairman), "ERUDIT Technical committee D on Traffic and Telecommunications: Application of soft computing Techniques to the telecommunication domain”, Aachen, Germany, Sept 1997.

[11] Y. C. Liu and C. Douligeris, Static vs. adaptive feedback congestion controller for ATM networks, IEEE Global Telecommunication Conference, GLOBECOM'95, Singapore, 1995
[12] D. Jensen, B-ISDN network management by a fuzzy logic controller, IEEE Global Telecommunications Conference, GLOBECOM '94, pages 799-804, 1994.

[13] C. Chang and R. Cheng, Traffic control in an ATM network using fuzzy set theory, IEEE INFOCOM'94 Conference, pp. 1200-1207, Toronto, Canada, June 1994.

[14] R-G. Cheng and C-J. Chang, Design of a fuzzy traffic controller for ATM networks, IEEE/ACM Transactions on Networking, 4(3), pp. 460-469, June 1996.

[15] A. Pitsillides, Y.A. Sekercioglu, and G. Ramamurthy, Fuzzy Backward Congestion Notification (FBCN) congestion control in Asynchronous Transfer Mode (ATM), IEEE Global Telecommunications Conference, GLOBECOM'95, pp. 280-285, Singapore, 1995.

[16] A. Pitsillides, Y.A. Sekercioglu, and G. Ramamurthy, Effective control of traffic flow in ATM networks using fuzzy logic based explicit rate marking (FERM), IEEE Journal on Selected Areas in Communications, 15(2), pp. 209-225, February 1997.

[17] B. Qiu. A predictive fuzzy logic congestion avoidance scheme, IEEE Global Telecommunications Conference, GLOBECOM'97, vol. 2, pp. 967-971, Society, 1997.

[18] M. Sugeno. Industrial Applications of Fuzzy Control. North-Holland, 1985.

[19] L. A. Zadeh. Fuzzy Logic. IEEE Computer. pp. 83-93, April 1988

[20] M. Sugeno and M. Nishida. Fuzzy Control of a Model Car. Fuzzy Sets and Systems, pp. 105-113, vol. 10, 1985.

[21] Q. Hu, D. W. Petr and C. Braun, Self-tuning fuzzy traffic rate control for ATM networks, IEEE International Conference on Communications, ICC'96, pp. 424-428, Dallas, Texas, USA, 1996. 